in electric lamp bulbs was of the order of $10^{-3} \mathrm{~mm}$. of mercury, while at the present day the residual gas pressure in certain valves can be as low as $10^{-9} \mathrm{~mm}$. of mercury. It is largely as a result of these improvements in vacuum technique and their influence on the 'longevity' of thermionic valves that projects such as that of trans-Atlantic telephone cables have become practicable. In the submerged repeaters of the present trans-Atlantic cable, there are some three hundred valves, and the success of the scheme depends upon these valves having lives of the order of twenty years. The development of the thermionic valve has provided the technical basis for the building up of an entirely new subject, namely, that of electronics.

The release, in useful form, of the energy of nuclear fission is, of course, the supremely important new application of physical science in the field of engineering. After describing the basic phenomena of the disruption of the uranium-235 nucleus and mentioning that the first self-sustaining pile generating heat by a nuclear fission chain was set up by Fermi in Chicago in 1942, Sir Edward stated that, in the nuclear energy station at present under construction in Cumberland, the reactor temperature would be maintained at about $400^{\circ} \mathrm{C}$. and that carbon dioxide gas under pressure would transfer heat from the uranium-graphite core to steam-raising equipment which would operate electrical generating equipment of normal type. Because of the temperature limitation in the reactor, however, it is unlikely that the overall efficiency of this station will exceed 25 per cent. Sir Edward went on to mention the principles of the 'breeder pile' and the problems associated with it. He then remarked: "You will have seen that the subject is now so far developed that much of the work is out of the hands of the physicist working alone. But I do want to stress that our nuclear engineer will be dealing with problems which, as an engineerbe he eivil, mechanical or electrical-he will never have encountered before. Materials will have to take on for him an entirely new meaning." There would be problems associated with materials hitherto unfamiliar in engineering practice, important because of their neutron-absorbing properties, together with exceedingly important metallurgical questions concerned with the development of materials for use at temperatures much higher than those which had been common in the past. "It seems to me," he went on, "that our interest in the nuclear energy field should be one of intense preoccupation. I see, ahead, the development of an enormous industry based on technology - the industry of not only supplying ourselves with nuclear electricity generating stations, but of supplying other countries with them and pos. sibly selling them fissile materials which we have ourselves produced from fertile uranium or thorium."

Remarking that his examples had so far been drawn from the realm of physies and engineering, Sir Edward turned to several instances of the co-operation of the chemist and the engineer. He took first that of the extraction of germanium from flue dust. This metal, which is now of immense importance as a semiconductor for the manufacture of diodes and triodes for the electronics industry, may be present in a proportion of the order of $0 \cdot 5-1 \cdot 0$ per cent in flue dust. A novel metallurgical treatment has been developed which is much more successful in extracting the metal from certain dusts than the ordinary acid treatment. Sir Edward then mentioned some of the problems associated with the manufacture of titanium and zirconium, the use of sodium benzoate as a corrosion inhibitor and finally, as an example of existing technological development facilitating the work of the pure scientist, the application of the technique of distillation to such laboratory processes as the fractionation of isotopes.

In his concluding remarks, Sir Edward referred to the importance of the development of automatic methods in the manufacturing industry. This is a revolutionary development which will call for statesmanship on the part of both management and labour. He also spoke with approval of the policy of the Atomic Energy Authority in respect of regarding industrial firms as the appropriate bodies to carry out the design as well as the construction of nuclear power plants. He regarded it as essential that nuclear scientific and engineering knowledge should be available to manufacturing firmis. The close and effective partnership of science and engineering is, in his opinion, something which is absolutely vital to the economic progress of Great Britain.

\section{NATIONAL PHYSICAL LABORATORY NOTES ON APPLIED SCIENCE}

$\mathrm{T}$

HE latest publications in the series "Notes on Applied Science" of the National Physical Laboratory, Teddington*, illustrate the wide range of investigations undertaken by the Laboratory, since all three are based on actual experience at the Laboratory. No. 10 in the series, "Noise Measurement Techniques", deals with the measurement of noise, defined as "any sound which is undesired by the recipient" ; and is intended to act as a guide to the choice of methods and techniques suitable for the measurement of the physical characteristics of noise in varying circumstances. In two introductory sections, the general, physical and subjective characteristics of noise and the principles of broad-band and narrow-band analysis are discussed, and the remainder of the booklet is devoted to a detailed description of noise-measuring equipment, its operation and calibration. Microphones, pre-amplifiers, output meters, recorders and analysers are given full and adequate treatment, and the booklet concludes with a brief, illustrated, account of the Mobile Acoustical Laboratory at Teddington.

The second booklet, No. 11, is concerned with wind effects on bridges and other flexible structures, and is based on wind tunnel research carried out in the Aerodynamics Division of the Laboratory, where extensive investigations have been made recently on the oscillatory effects of winds on models of two proposed suspension bridges-the Severn Bridge between Beachley and Aust, and the Runcorn-Widnes Bridge over the River Mersey. Most of the booklet is devoted to oscillatory effects although brief reference is made also to static wind loads; while bridges are the primary concern of the booklet, much of the content is also applicable to other flexible structures, such as tall chimney stacks, transmission lines and buildings. It is emphasized that an examination of the history of bridge failures reveals that a bridge

* National Physical Laboratory: Notes on Applied Science. No. 10: Noise Measurement Techniques. Pp. iv $+40+3$ plates; 28 . net No. 11: Wind Effects on Bridges and other Flexible structures Pp. iv $+14+7$ plates ; 1s. 6d. net. No. 12 : Calibration of TemperatureH.M.S.O., 1955.) 
fails because of both static action of wind (that is, a tendency to give the bridge a steady deflexion) and dynamic action (that is, a tendency to set the bridge oscillating), and that, in addition, the dynamic effects can occur in two ways, termed aerodynamic instability and buffeting. The experimental methods to investigate static action, aerodynamic instability, and buffeting on bridges are described in detail and the necessary equipment and the models used at the Laboratory are illustrated.

The third booklet, No. 12, entitled "Calibration of Temperature-Measuring Instruments", refers to a different but important section of the National Physical Laboratory's work, namely, the maintenance of standards, and in this particular case to the basic scales of temperature and the instrumental means by which they are realized. It is the most up-to-date text on the subject, and should receive the widest possible circulation. It sets forth clearly what is meant by a temperature scale, and draws a careful distinction between accuracy and reproducibility in temperature measurement, quoting as an example that the sulphur boiling point, defined as $444 \cdot 600^{\circ} \mathrm{C}$., may be reproduced with the platinum-resistance thermometer to about $0.005 \mathrm{deg}$. C., but its value by gas-thermometer observation is not known to better than $\pm 0 \cdot 1$ deg. $C$. The various arrangements for the realization of the primary fixed points, including the triple point of water, and the necessary precautions to be taken, are described in detail, in addition to the primary standard and working standard instruments used at the Laboratory. One section is devoted to a description of the methods used for the routine testing of temperature-measuring instruments, and another to the baths and furnaces used in comparison tests with standard instruments ; in an appendix, the definition of the 1948 International Temperature Scale is reproduced.

\title{
METABOLISM OF PROPIONIC ACID IN ANIMAL TISSUES
}

\author{
By Dr. MARTIN FLAVIN*, PRISCILLA J. ORTIZ and PROF. SEVERO OCHOA \\ Department of Biochemistry, New York University College of Medicine, New York
}

\begin{abstract}
$\mathrm{A}$ NIMAL tissues can oxidize propionic acid; but the mechanism of this oxidation has long remained obscure. Some workers believe that this fatty acid undergoes $\alpha$-oxidation to yield acrylic, lactic and pyruvic acid ${ }^{1-3}$, the latter being oxidized to carbon dioxide and water via the citric acid cycle. However, experiments on the distribution of carbon14 from labelled propionate in tissue glycogen ${ }^{4}$ and acetyl groups ${ }^{5}$, or in lactate ${ }^{8}$, indicated the occurrence of a symmetrical intermediate between propionate and pyruvate, such as would be formed if propionate or acrylate were first carboxylated to succinate or fumarate, followed by oxidation of the dicarboxylic acid through the citric acid cycle. This possibility appeared to be strengthened by the finding of bacterial enzymes which catalyse the reverse reaction, that is, the decarboxylation of succinate via succinyl coenzyme A, to propionate? More recently, an adenosine triphosphate-dependent conversion of propionate to succinate by fixation of carbon dioxide has, in fact, been reported to occur in liver extracts ${ }^{8}$.
\end{abstract}

\section{Biosynthesis of Methylmalonate}

We have found that dialysed extracts and ammonium sulphate fractions of pig heart, supplemented with propionyl coenzyme A, adenosine triphosphate and magnesium ions, fix carbon-14 dioxide to form a compound easily hydrolysed by alkali to yield carboxyl-labelled methylmalonic (isosuccinic) acid ${ }^{9}$. The latter has been identified by $(a)$ paper chromatography and autoradiography, (b) recrystallization with carrier, and $(c)$ recovery of radioactivity in a derivative, C-methylbarbituric acid, or in propionic acid and carbon dioxide following decomposition at $150^{\circ}$. Under optimal conditions for the synthesis of methylmalonate, no succinate is formed by the pig heart preparations. The conversion of propionate to

* Postdoctoral fellow of the American Heart Association. Most of this work was done while the author was assigned from the Laboratory Health, U.S. Public Health Service. methylmalonate has been independently observed by Katz and Chaikoff ${ }^{10}$ in rat liver slices.

The requirements for methylmalonate synthesis are shown in Table 1. It may also be seen in the table that propionyl coenzyme A can be replaced, although less effectively, by a mixture of propionate and coenzyme A. Under these conditions, propionyl coenzyme $\mathbf{A}$ is undoubtedly formed through reaction $\mathbf{1}$, catalysed by the acetate activating enzyme ${ }^{11,12}$, which is also active with propionate.

Propionate $+\mathrm{CoA}+\mathrm{ATP} \rightleftharpoons$ Propionyl $\mathrm{CoA}+$ $\mathrm{AMP}+\mathrm{PP}$

Little or no fixation (or exchange) of carbon-14 dioxide was observed with acrylyl or succinyl coenzyme $\mathbf{A}$ in place of propionyl coenzyme $\mathrm{A}$, or with succinate or

Table 1. Requirements For Enzymatic Synthesis of MethyLMALONATE FROM CARBON-14 DTOXIDE AND PROPIONYL COENZYME A

\begin{tabular}{|c|c|c|c|c|}
\hline \multicolumn{4}{|c|}{ Components } & $\begin{array}{l}\text { Carbon-14 } \\
\text { dioxide fixed } \\
\text { (per cent)* }\end{array}$ \\
\hline \multicolumn{4}{|c|}{ Complete system $\uparrow$} & $6 \cdot 0$ \\
\hline \multicolumn{4}{|c|}{ No enzyme " + coenzyme A } & $6 \cdot 0$ \\
\hline \multirow{2}{*}{\multicolumn{4}{|c|}{ No adenosine triphosphate }} & $0 \cdot 03$ \\
\hline \multirow{2}{*}{\multicolumn{4}{|c|}{ No propionyl coenzyme A }} & 0 \\
\hline & & & & 0.02 \\
\hline ," & ," & ," & propionate & $1 \cdot 5$ \\
\hline ", & ," & $"$, & methylmalonate & $0 \cdot 1$ \\
\hline & ", & & succinate & 0.02 \\
\hline$"$, & 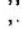 & & succinyl coenzyme $\mathbf{A}$ & 0.01 \\
\hline
\end{tabular}

* Results from several experiments.

+ Consisting of following components (in $\mu$ moles): tris(hydroxymethyl) aminomethane buffer, $p$ B $7 \cdot 0,50$; magnesium chloride, 2 ; reduced glutathione, 5 ; adenosine triphosphate, 2 ; propionyl coenzyme A, 1 ; potassium bicarbonate- ${ }^{-14} \mathrm{C}, 10$; pig heart extract with 5 mgm protein. Volume, $1.0 \mathrm{ml}$. Other additions, each $1 \mu$ mole except succinyl coenzyme A, 2 moles. Cocnzyme A added in the last four experiments. Incubation $60 \mathrm{~min}$. at $25^{\circ}$. Heart and other extracts prepared by homogenizing fresh tissue in a Waring blendor
with 4 volumes of $0.05 M$ potassium phosphate buffer, $p H{ }^{\circ}, 4$ with 4 volumes of $0.05 M$ potassium phosphate buffer, $p \mathrm{H} 7 \cdot 4$, containing $0.2 M$ potassium chloride, and centrifuging 15 min. at phosphate buffer, $p \mathrm{H} 7 \cdot 0$, containing $0.001 M$ neutralized L-cysteine. phosphate buffer, $p H 7.0$, containing $0.001 M$ neutralized $L$-cysteine. extracts by precipitation with ammonium sulphate between 30 and 50 per cent saturation, and dialysed as above, have also been used. 